\title{
Redução dos impactos ambientais $\epsilon$ aumento da pontuação LEED através de melhorias no concreto
}

\begin{tabular}{c}
\hline GRAZIELLE RIBEIRO VICENTE - ENGENHEIRA CIVIL \\
\hline SELMO CHAPIRA KUPERMAN - DIRETOR \\
\hline EDUARDO ISSAMU FUNAHASHI JÚNIOR - ENGENHEIRO CIVIL \\
DESEK
\end{tabular}

$\frac{\frac{\text { HENRIQUE MACHADO DE MENDONÇA - GERENTE }}{\text { CAIO ALENCAR RIBEIRO - ANALISTA DE SUSTENTABILIDADE }}}{\text { EToOL }}$

\section{RESUMO}

A BUSCA POR CONSTRUÇÕES MAIS SUSTENTÁVEIS TEM AUMENTADO NOS ÚLTIMOS ANOS. UMA FORMA DE AVALIAR OS IMPACTOS AMBIENTAIS ASSOCIADOS A TODAS AS ETAPAS DA VIDA DE UM PRODUTO OU SERVIÇO É A CHAMADA AvaliaçÃo de Ciclo de Vida (ACV), com CARActerísticas SUSTENTÁVEIS ASSEGURADAS VIA CERTIFICADO LEED. QUANTO MAIOR A PONTUAÇÃO LEED DE UMA EDIFICAÇÃO, MAIOR É A REDUÇÃO DOS SEUS IMPACTOS AMBIENTAIS, PODENDO CHEGAR A 4 PONTOS PARA O CRÉDITO DE ACV, O QUE REPRESENTA UMA REDUÇÃO DE IMPACTO DE 20\%.
COMO O CONCRETO É UM DOS MATERIAIS MAIS CONSUMIDOS NO MUNDO, FOI FEITO UM ESTUDO ABRANGENDO ALGUMAS ALTERAÇÕES NA SUA COMPOSIÇÃO QUE CONTRIBUÍRAM SIGNIFICATIVAMENTE PARA A REDUÇÃO DOS IMPACTOS AMBIENTAIS E PARA O AUMENTO DA PONTUAÇÃO LEED DA OBRA. AS ALTERAÇÕES FEITAS NOS TRAÇOS DE CONCRETO PERMITIRAM A OBTENÇÃO DE 3 PONTOS NA CERTIFICAÇÃO LEED, ALÉM DISSO PERMITIRAM A MITIGAÇÃO DE MANIFESTAÇÕES PATOLÓGICAS NAS ESTRUTURAS DE CONCRETO E CONTRIBUÍRAM PARA A REDUÇÃO DO CUSTO DA OBRA.

Palavras-chave: sustentabilidade, LEED, impacto ambiental, ACV, concreto.

\section{OBJETIVO}

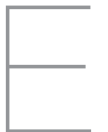
ste trabalho apresenta um estudo realizado em um edifício em construção na cidade de São Paulo, com o objetivo de reduzir os impactos ambientais causados pela construção civil e, consequentemente, aumentar a pontuação LEED, por meio da alteração dos materiais constituintes do concreto.

\section{INTRODUÇÃO}

Devido ao grande consumo de recursos naturais e emissões poluentes causados pela construção civil, há uma crescente necessidade de redução dos impactos ambientais e bus- ca por alternativas sustentáveis neste setor. Grande parte destes impactos vem da etapa da produção dos materiais utilizados na construção civil e um dos materiais que se destaca é o concreto.

O concreto é o segundo material mais consumido no mundo e, por este motivo, alternativas para melhoria deste material contribuem significativamente para tornar a construção mais sustentável.

Uma forma de avaliar os impactos ambientais associados a todas as etapas da vida de um produto ou serviço é a chamada Avaliação de Ciclo de Vida (ACV), que é regulada pelo padrão internacional ISO 14044 (e EN15978 especificamente para edificações) e sua aplicação na área de construção civil é utilizada mundialmente para promover desenvolvimento sustentável.

A ACV foi utilizada para quantificar e analisar a contribuição dos impactos ambientais de uma edificação comercial no Brasil, que possui características sustentáveis asseguradas via certificado LEED. As fontes de dados de entrada são provenientes da documentação do projeto, principalmente o projeto estrutural e lista de materiais com quantitativo e especificações.

O crédito do LEED como estudo 
de ACV pode atingir até 4 pontos e tem o intuito de medir o desempenho ambiental de produtos e materiais utilizados, analisando a estrutura, a fundação e a envoltória, com foco nos materiais, bem como estudando como se pode diminuir os impactos negativos causados ao meio ambiente.

Esses 4 pontos para o crédito de ACV são divididos da seguinte forma:

> 1 ponto - mínimo garantido por realizar o estudo de ACV;

> 2 pontos - 5 \% de redução de impacto;

3 pontos - 10\% de redução de impacto;

$>4$ pontos - 20\% de redução de impacto.

Quando o estudo de ACV é feito na obra, já se garante 1 ponto na certificação LEED e os demais pontos são obtidos com o resultado de mudanças implementadas para diminuir os impactos globais da edificação.

Este trabalho mostra como algumas alterações nos traços de concreto desta obra contribuíram para a redução dos impactos ambientais e o aumento da pontuação LEED da edificação.

\section{AVALIAÇÃO DO CICLO DE VIDA DA OBRA}

O estudo de Avaliação do Ciclo de Vida da edificação em questão foi feito com o uso do software eToolLCD. Inicialmente, foi necessário detalhar as melhorias que foram implantadas no projeto, incluindo uma estimativa de redução no consumo de materiais quando comparado com práticas atuais de mercado ou com o projeto básico. Por exemplo: laje protendida comparada com laje maciça permite uma redução de $\mathrm{X} \%$ de concreto e uma redução de $Y \%$ de aço. Esse tipo de informação é apresentado como resultado do estudo.

O cálculo do potencial de aquecimento global inicial foi realizado a partir dos traços de concreto advindos da concreteira. Em seguida, procedeu-se o estudo de dosagem com vistas a reduzir o consumo de cimento e a utilizar materiais mais sustentáveis como, por exemplo, o cimento composto com alto teor de escória granulada de alto forno. Por fim, calculou-se o potencial de aquecimento global com os novos traços de concreto.

No estudo inicial, estimou-se que, ao longo do período de 60 anos, a emissão de $\mathrm{CO}_{2}$ seria de aproximadamente 30.000 toneladas, o que equivale a cerca de 150.000 árvores para
Tabela 1 - Cálculo inicial dos impactos ambientais da edificação

Soma do Potencial de Aquecimento Global (PAG) em kg CO 2 e por materiais

Torre

Total

Nome dos elementos

Concrete I Unreinforced I Blast Furnace Slag Blends | $40 \mathrm{MPa} \mid 10 \% \mathrm{BFS}$

$\begin{array}{crr}\text { Concrete I Unreinforced I Portland Cement Blends | } 30 \mathrm{MPa} & 5,395,587.30 & 5,395,587.30 \\ \text { Concrete I Unreinforced I Portland Cement Blends | } 40 \mathrm{MPa} & 3,448,111.15 & 3,448,111.15 \\ \text { Cementitious Binders | Mortars and Renders | 1 cement : } 4 \text { sand } & 3,416,646.17 & 3,416,646.17 \\ \text { Concrete I Unreinforced I Blast Furnace Slag Blends | } & 2,515,701.84 & 2,515,701.84 \\ \text { 50 MPa | 10\% BFS } & \end{array}$

Concrete | Unreinforced | Portland Cement Blends I Unspecified ～1,697,405.04 1,697,405.04

Ferrous Metals I Steel I Reinforcement bar I Unspecified $\quad 1,104,119.89 \quad 1,104,119.89$

Concrete | Unreinforced I Blast Furnace Slag Blends | $30 \mathrm{MPa} \mid 10 \% \mathrm{BFS}$

$759,522.24 \quad 759,522.24$

Metals (Non-Ferous) | Aluminium Unspecified

Plastics | Acrylic I Unspecified

$342,232.86 \quad 342,232.86$

$236,242.93 \quad 236,242.93$

Glazing | Glass and Films | Flat Glass

$212,641.53 \quad 212,641.53$

Paints and Finishes I Unspecified I 1 Coat

$129,628.42 \quad 129,628.42$

Metals (Non-Ferous) I Aluminium Extruded

$83,990.78$

$83,990.78$

Glazing | Windows | Aluminium Framed | No Thermal Break | Single Glaze I Domestic Fixed

$80,035.84 \quad 80,035.84$

Ferrous Metals I Steel I General I Unspecified

Glazing | Windows | Timber Framed I Double Glaze | Domestic 50\% Opening

$37,354.44 \quad 37,354,44$

$15,984.76 \quad 15,984.76$

Plastics I High Density Polyethylene (HDPE) I Unspecified

Ferrous Metals I Steel I Stainless I Unspecified

$14,820.26 \quad 14,820.26$

Plaster and Mineral Derived Products | 100\% Primary Gypsum I Plaster I Unspecified

Rubber I Synthetic

$9,582.82$

$9,582.82$

$7,970.57$

$7,970.57$

Resins and Adhesives I Mastic Sealant

$5,169.80$

$5,169.80$

Resins and Adhesives I Mastic Sealant

25.41

25.41

Timber I Sustainably Sourced I General I Unspecified

Timber I Sustainably Sourced I Plywood I Unspecified

$-9,259.54$

$-9,259.54$

$-69,531.79$

$-69,531.79$ 
fazer a compensação, como mostra a Tabela 1.

Em decorrência desta grande emissão de $\mathrm{CO}_{2}$, a diminuição dos impactos causados pelo concreto foi um dos focos principais deste estudo.

De acordo com a NBR 16697/2018, o teor de escória do CP II E pode variar de 6 a 34\% e do CP III de 35 a 75\%. De acordo com o relatório de ensaio do cimento fornecido para esta obra, o cimento CP III 40 RS apresentou teor de escória igual a 44\%.

A escória de alto forno é um resíduo do processo siderúrgico, resultante da fabricação do ferro gusa. Sua adição na fabricação do cimento, em substituição ao clínquer, contribui para a redução dos impactos ambientais, pois evita a queima de combustível fóssil e a descarbonatação do calcário relativas à produção de clínquer. Por este motivo foi recomendado o uso do cimento CP III em algumas estruturas desta obra e, com isso, já foi possível observar uma melhoria significativa no estudo de redução do impacto ambiental.
A alteração do tipo de cimento possibilitou uma redução total de 9,78\% na emissão de $\mathrm{CO}_{2}$ para o escopo em análise, que representa 2.934 toneladas de $\mathrm{CO}_{2}$, equivalente a aproximadamente 14.670 árvores para fazer a compensação, de acordo com o requisito do crédito LEED. Esta redução não se refere a edificação como um todo, incluindo energia e outros elementos construtivos e instalações, por exemplo.

Com essas melhorias, o projeto alcançou 12\% de redução de impacto em GWP e 10\% em outros 2 indicadores, garantindo os 3 pontos na certificação LEED, como mostra Figura 1.

Todas as melhorias aplicadas ao projeto tiveram uma relevância maior no Potencial de Aquecimento Global (GWP), a principal delas foi o uso do cimento com elevado teor de escória de alto forno, que também ajudou a minimizar os impactos de outros indicadores, como o potencial de acidificação do solo e da água (AP) e o potencial de criação fotoquímica de

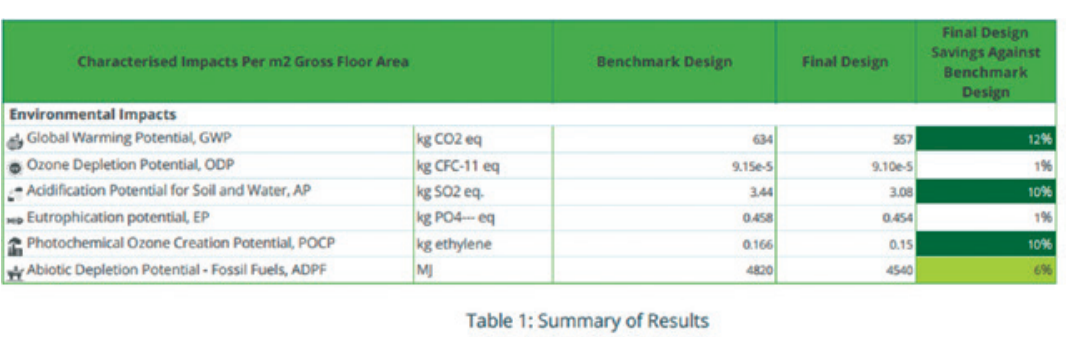

The Final Design shows an expected performance improvement against Business as Usual for 6 of the 6 environmental indicators. The following low impact strategies are included in the Final Design:

\begin{tabular}{|c|c|c|c|c|c|c|}
\hline Design Strategy Performance & GWP & $\begin{array}{c}\text { OOP } \\
\text { OD }\end{array}$ & $\stackrel{\circ}{A P}$ & $\begin{array}{l}H \rightarrow \\
E P\end{array}$ & $\underset{\text { POCP }}{\text { if }}$ & $\frac{\text { fir }}{A D P F}$ \\
\hline Foundation elements optimization & $0.33 \%$ & $0.07 \%$ & $0.34 \%$ & 0.236 & 0.428 & $0.34 \%$ \\
\hline Prestressed slab & 0.655 & $0.13 \mathrm{~m}$ & 0.600 & 0.356 & $0.60 \mathrm{~m}$ & $0.60 \%$ \\
\hline Auditorium Exposed concrete wall & $0.10 \%$ & $0.05 \%$ & $0.08 \%$ & 0.156 & $0.08 \%$ & $0.12 \%$ \\
\hline Steel recycled content Gerdau & 0.026 & $0.00 \%$ & $0.00 \%$ & -00048 & 0.026 & $0.01 \%$ \\
\hline Steel Recycle Content ArcelorMittal & 1.154 & 0.075 & $0.24 \%$ & $228 \mathrm{M}$ & 1.475 & $0.61 \%$ \\
\hline Concrete downcycling & 0.144 & $0.28 \mathrm{~m}$ & $0.14 \%$ & $0.23 \%$ & $0.12 \%$ & $0.59 \%$ \\
\hline Cement CP III 44\% BFS & $9.786 \%$ & & $9.01 \%$ & 2.1856 & 7.270 & $3.65 \%$ \\
\hline
\end{tabular}

Figura 1

Cálculo dos impactos ambientais da edificação ozônio (POCP). A certificação LEED pede que para obter 3 pontos é necessário reduzir 10\% dos impactos em 3 indicadores, sendo um deles obrigatoriamente o GWP.

\section{MELHORIAS NO CONCRETO DA OBRA}

Durante a fase inicial da obra, foram feitos estudos prévios de dosagem com o objetivo de reduzir o consumo de cimento, mitigar manifestações patológicas e utilizar materiais mais sustentáveis. Os estudos de dosagem foram feitos com base nas especificações de projeto para os seguintes elementos estruturais:

Blocos de fundação: $f_{c k} \geq 40 \mathrm{MPa}$, $\mathrm{E}_{\mathrm{cs}} \geq 32 \mathrm{GPa}$ para tensão de $14 \mathrm{MPa}$ e relação a/c $\leq$ 0,55;

Lajes protendidas dos subsolos: $f_{0}$ 7 dias $\geq 35 \mathrm{MPa}, \mathrm{f}_{\mathrm{ck}} \geq 40 \mathrm{MPa}, \mathrm{E}_{\mathrm{cs}}$ $\geq 32$ GPa para tensão de $14 \mathrm{MPa}$ e relação $a / c \leq 0,55$;

- Paredes de concreto aparente: $\mathrm{f}_{\mathrm{ck}} \geq 40 \mathrm{MPa}, \mathrm{E}_{\mathrm{cs}} \geq 32 \mathrm{GPa}$ para tensão de $14 \mathrm{MPa}$ e relação $\mathrm{a} / \mathrm{c} \leq$ 0,55 ;

- Elementos estruturais do $3^{\circ}$ subsolo ao $5^{\circ}$ pavimento: $f_{c k} \geq 40 \mathrm{MPa}$, $\mathrm{E}_{\mathrm{cs}} \geq 32 \mathrm{GPa}$ para tensão de 14 MPa e relação a/c $\leq 0,55$;

- Pilares da fundação ao pavimento $0: f_{c k} \geq 50 \mathrm{MPa}, E_{c s} \geq 37 \mathrm{GPa}$ para tensão de $18 \mathrm{MPa}$ e relação $\mathrm{a} / \mathrm{c} \leq$ 0,55 ;

- Elementos estruturais do $6^{\circ}$ pavimento à cobertura: $f_{c k} \geq 35 \mathrm{MPa}$, $\mathrm{E}_{\mathrm{cS}} \geq 29 \mathrm{GPa}$ para tensão de 12 $\mathrm{MPa}$ e relação $\mathrm{a} / \mathrm{c} \leq 0,55$;

- Reservatório: $\mathrm{f}_{\mathrm{ck}} \geq 40 \mathrm{MPa}, \mathrm{E}_{\mathrm{cs}} \geq$ 32 GPa para tensão de $14 \mathrm{MPa}$ e relação $\mathrm{a} / \mathrm{c} \leq$ 0,55.

No caso dos blocos de fundação, o uso do cimento CP III foi recomendado, 
não somente para a redução dos impac- creto durante o processo de hidratação formação de etringita tardia (DEF), que tos ambientais, mas também para a re- do cimento, o que contribui para mitigar a podem comprometer a segurança e dudução das temperaturas internas do con- formação de fissuras de origem térmicaea rabilidade das estruturas.

\section{Tabela 2 - Melhorias nos traços de concreto da obra}

Consumo $\left(\mathrm{kg} / \mathrm{m}^{3}\right)$

\begin{tabular}{|c|c|c|c|c|c|c|c|c|c|c|c|c|c|c|c|c|c|c|}
\hline 4 & मु & 总妾 & 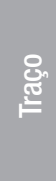 & $\begin{array}{l}\text { 응 } \frac{\text { ㅁ }}{10} \\
\text { 을 흥 }\end{array}$ & 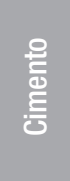 & 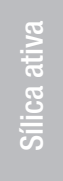 & 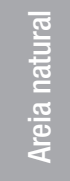 & 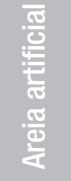 & 号 & 焉 & 哛 & 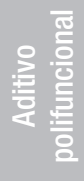 & 竞变 & 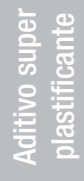 & 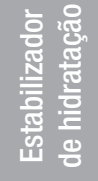 & 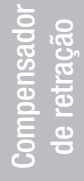 & 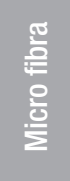 & 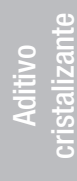 \\
\hline \multirow{2}{*}{40} & \multirow{2}{*}{32} & \multirow{2}{*}{ 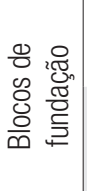 } & 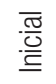 & 항 & 394 & - & 219 & 511 & - & 1015 & 188 & 3,94 & - & - & - & - & - & - \\
\hline & & & $\overline{\stackrel{\widetilde{\varpi}}{\frac{1}{4}}}$ & 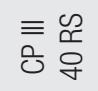 & 394 & - & 219 & 511 & - & 1015 & 188 & 3,94 & - & - & - & - & - & - \\
\hline \multirow{2}{*}{40} & \multirow{2}{*}{32} & \multirow{2}{*}{ 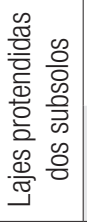 } & 制 & 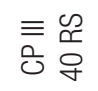 & 380 & 27 & 228 & 532 & - & 1045 & 170 & & 1,90 & - & - & 10 & 0,6 & - \\
\hline & & & 휸 & $\underset{\overline{0}}{\stackrel{w}{0}}$ & 340 & 27 & 239 & 557 & - & 1045 & 170 & 3,40 & 1,02 & - & 0,734 & 10 & 0,6 & - \\
\hline \multirow[b]{2}{*}{40} & \multirow[b]{2}{*}{32} & \multirow{2}{*}{ 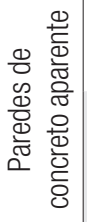 } & $\frac{\overline{\underline{\underline{0}}}}{\underline{\underline{\underline{\underline{E}}}}}$ & 竞 & 420 & - & 507 & 217 & 152 & 863 & 180 & 3,36 & 1,26 & - & 0,63 & 10 & - & - \\
\hline & & & $\overline{\text { शृ }}$ & 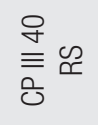 & 360 & 23 & 566 & 377 & 508 & 339 & 180 & 2,88 & 1,08 & 1,70 & 0,54 & 10 & - & - \\
\hline \multirow{2}{*}{40} & \multirow{2}{*}{32} & \multirow{2}{*}{ 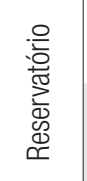 } & $\frac{\overline{\underline{\sigma}}}{\stackrel{\underline{\underline{O}}}{\underline{\underline{E}}}}$ & 응 & 420 & - & 507 & 217 & 152 & 863 & 180 & 3,36 & 1,26 & - & 0,63 & 10 & - & 3,29 \\
\hline & & & 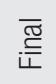 & 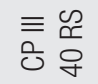 & 360 & 23 & 566 & 377 & 508 & 339 & 180 & 2,88 & 1,08 & 1,70 & 0,54 & 10 & & 2,88 \\
\hline \multirow[b]{2}{*}{35} & \multirow[b]{2}{*}{29} & \multirow{2}{*}{ 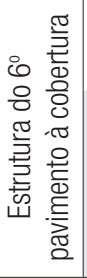 } & 画 & 흥 움 & 349 & - & 230 & 536 & 152 & 859 & 188 & 3,49 & - & - & - & - & - & \\
\hline & & & 휸 & 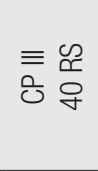 & 345 & - & 229 & 534 & 153 & 866 & 190 & 2,76 & - & - & - & - & - & \\
\hline \multirow[b]{2}{*}{40} & \multirow[b]{2}{*}{32} & \multirow{2}{*}{ 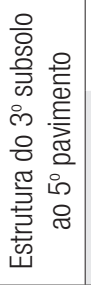 } & 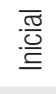 & 응 움 & 375 & - & 221 & 516 & 153 & 866 & 190 & 3,00 & - & - & - & - & - & 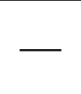 \\
\hline & & & 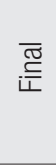 & 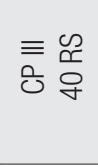 & 375 & - & 221 & 516 & 153 & 866 & 190 & 3,00 & - & - & - & - & - & \\
\hline \multirow{2}{*}{50} & \multirow{2}{*}{37} & \multirow{2}{*}{ 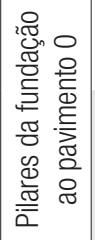 } & 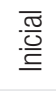 & 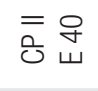 & 462 & - & 273 & 409 & 0 & 1026 & 176 & 3,23 & - & - & - & - & - & - \\
\hline & & & 휸 & 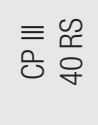 & 430 & - & 207 & 482 & 153 & 866 & 190 & 3,44 & - & - & - & - & - & \\
\hline
\end{tabular}


Para a realização do estudo térmico dos blocos de fundação, foram feitas simulações computacionais tridimensionais por meio do método dos elementos finitos, para avaliar as evoluções de temperaturas de alguns pontos da massa do concreto e consequentes tensões de tração oriundas do resfriamento do material. Como resultado deste estudo, foram definidas as temperaturas de lançamento do concreto e o plano de execução com menor risco de fissuração.

No caso das lajes protendidas do subsolo, como foi especificada resistência inicial superior a $35 \mathrm{MPa}$ aos 7 dias, devido à protensão do concreto, adotou-se o cimento CP II, pois este apresenta maiores resistências nas idades iniciais se comparado ao cimento CP III. Para os demais elementos estruturais, foram feitos estudos de dosagens comparando concretos com uso do cimento CP II e do cimento $\mathrm{CP}$ III.
Os estudos de dosagem para definição dos diferentes traços de concreto foram feitos com o objetivo de reduzir o consumo de cimento, focando na redução do impacto ambiental. Normalmente o uso do cimento CP III no concreto em comparação ao cimento CP II exige maior consumo para obter a mesma resistência de projeto, porém nos estudos de dosagem feitos para esta obra, foi possível perceber que os concretos com os dois tipos de cimento apresentaram resistências à compressão praticamente iguais, o que resultou na escolha do CP III para boa parte dos elementos estruturais, favorecendo ainda mais a pontuação do LEED e a redução dos impactos ambientais, devido ao emprego de cimentos com maior teor de escória de alto forno.

No concreto protendido das lajes dos subsolos, se fosse utilizado o cimento CP III seria necessário aumentar o consumo de cimento para atender à resistência inicial especificada

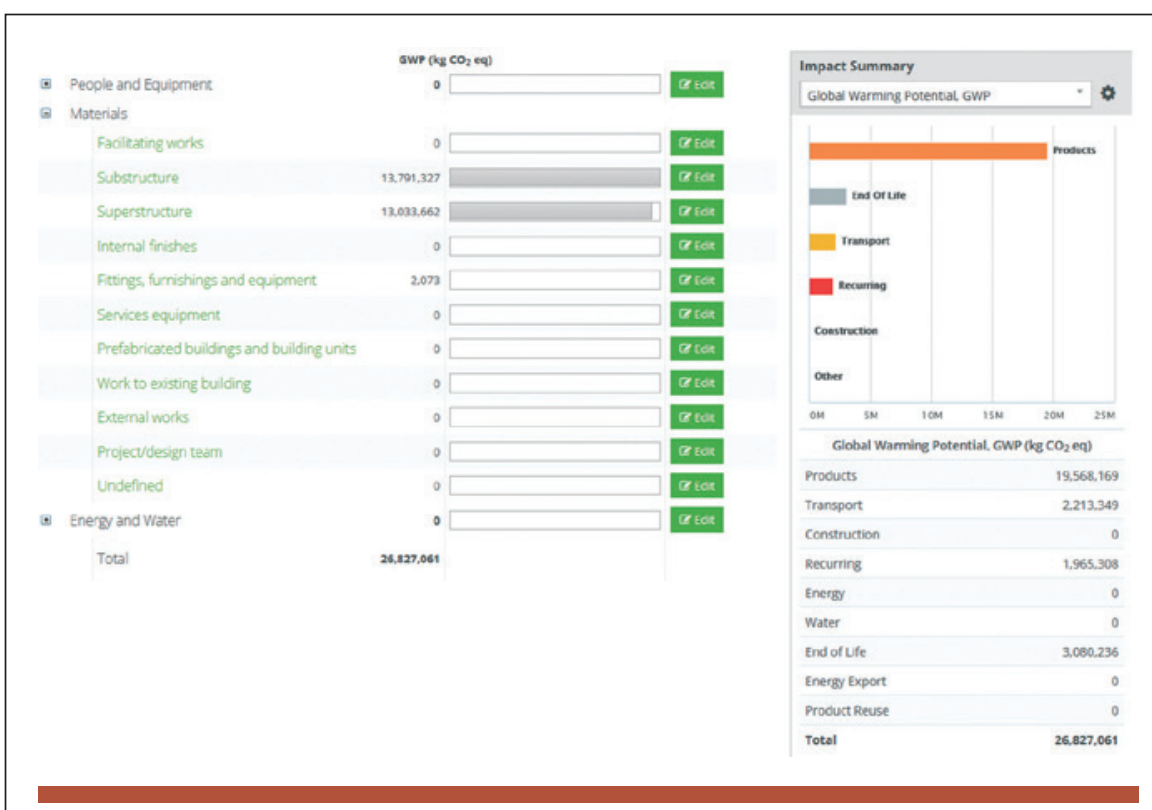

Figura 2

Resultado final do software eToolLCD em projeto. Neste caso, foi utilizado o cimento CP II e foi adicionada sílica ativa ao concreto, como substituição parcial do cimento, com o objetivo de reduzir o seu consumo no traço.

A sílica ativa, além de melhorar a qualidade do concreto, diminui o consumo de cimento e contribui para a redução do impacto ambiental, reduzindo a emissão de $\mathrm{CO}_{2}$, pois é um resíduo industrial.

A Tabela 2 apresenta os traços de concreto estudados pela concreteira, que foram chamados de "Inicial", e os traços de concreto ensaiados com o objetivo de mitigar manifestações patológicas e reduzir os impactos ambientais, que foram chamados de "Final", pois esses foram os traços de concreto utilizados na execução das estruturas.

Os traços considerados como iniciais representam as dosagens sem as melhorias propostas. Durante as dosagens experimentais no laboratório da concreteira, foram feitas alterações de modo a obter concretos com melhores desempenhos, seja no atendimento às especificações de projeto, seja para melhor tratar a questão da sustentabilidade.

Com base nestas melhorias nos traços de concreto, foi possível fazer os cálculos para definição da pontuação LEED e o projeto atingiu os requisitos do crédito para obtenção de 3 pontos no total.

A Figura 2 apresenta o resultado final do software eToolLCD após as melhorias no concreto da obra.

O uso do cimento CP III contribuiu para a diminuição do impacto incorporado do concreto, garantindo a viabilidade dos outros 2 pontos na certificação LEED desta obra. 


\section{CONCLUSÕES}

Este estudo mostrou a importância da escolha dos materiais constituintes do concreto para a redução dos impactos ambientais na fase inicial de projeto. As alterações feitas nos traços de concreto permitiram a obtenção de 3 pontos na certificação LEED desta obra, com redução de impacto maior que 10\% em pelo menos três indicadores de desempenho, incluindo o Potencial de Aquecimento Global.

Além da redução dos impactos ambientais, as alterações nos traços de concreto desta obra permitiram a mitigação de eventuais manifestações patológicas, tais como: fissuração de origem térmica e formação de etringita tardia nos blocos de fundação; fissuração causada pela retração plástica e/ ou por secagem nas lajes protendidas dos subsolos, paredes de concreto aparente e reservatório; entre outras.

Além do atendimento das especificações de Projeto, estas melhorias nos traços de concreto trouxeram benefícios para a construtora, tais como:

$>$ Redução do consumo de cimen- to, através de estudos prévios de dosagem;

> Redução de manifestações patológicas nas estruturas de concreto;

> Redução do custo da obra;

> Diferencial de mercado e inovação;

> Responsabilidade ambiental com a obtenção de 3 pontos na certificação LEED.

Ressalta-se que essas melhorias foram possíveis pois os estudos de dosagem e de Avaliação do Ciclo de Vida da edificação foram feitos nas fases iniciais do projeto.

\section{REFERÊNCIAS BIBLIOGRÁFICAS}

[1] EN 15978. Sustainability of construction works - Assessment of environmental performance of buildings - Calculation method. BSI, 2011.

[2] ISO 14044. Gestão ambiental - Avaliação do ciclo de vida - Requisitos e orientações. ABNT, 2014.

[3] NBR 16697. Cimento Portland - Requisitos. ABNT, 2018.

\section{Sistemas de Fôrmas para Edifícios}

Recomendações para a melhoria da qualidade e da produtividade com redução de custos

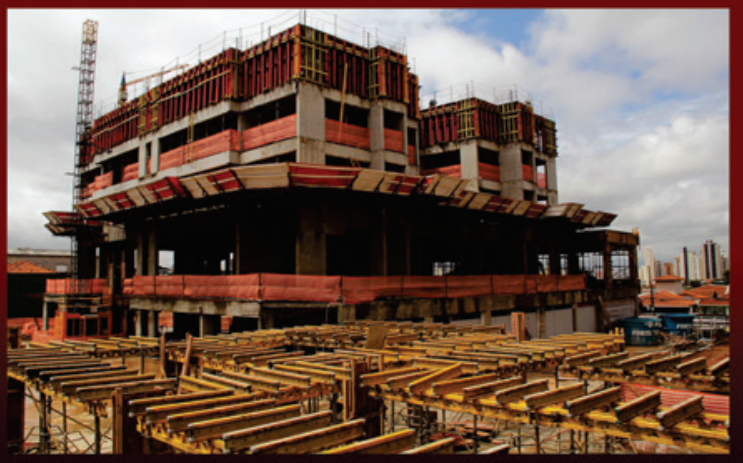

ANTONIO CARLOS ZORZI

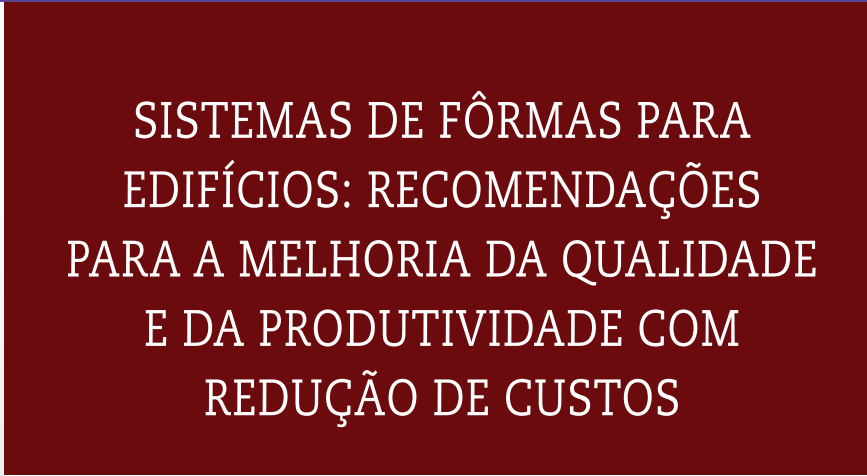

Autor: Antonio Carlos Zorzi

O livro propõe diretrizes para a racionalização de sistemas de fôrmas empregados na execução de estruturas de concreto armado e que utilizam o molde em madeira

As propostas foram embasadas na vasta experiência do autor, diretor de engenharia da Cyrela, sendo retiradas de sua dissertação de mestrado sobre o tema.

\section{DADOS TÉCNICOS}

ISBN 9788598576237

Formato: $18,6 \mathrm{~cm} \times 23,3 \mathrm{~cm}$

Páginas: 195

Acabamento: Capa dura

Ano da publicação: 2015
Patrocínio

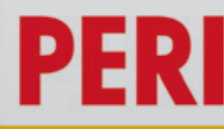

Aquisição: www.ibracon.org.br (Loja Virtual) 